\title{
High-Sensitive Troponin-T as a Predictive Outcome Factor in COVID-19 Hospitalised Patients: Analysis After One-Year Follow-Up
}

\author{
Darko Stojanović, Živko Ćetojević, ${ }^{1}$ Boris Dujaković, ${ }^{2}$ Mirko Stanetić, ${ }^{1,3}$ \\ Tamara Kovačević-Preradović, ${ }^{1,3}$ Bojan Stanetić, 2
}

\begin{abstract}
Introduction: Since December 2019, the humanity is constantly under affection of the severe acute respiratory syndrome coronavirus 2 (SARS-CoV-2). Despite global dissemination, neither the treatment or the specific predictive factors have been found or strictly defined yet.

Aim: Aim of this study was to assess the long-term (1 year) predictive value of high-sensitive Troponin T (hsTnT) in COVID-19 affected, hospitalised patients.

Methods: Between 5 March 2020 and 31 March 2020, 87 consecutive patients hospitalised at University Clinical Centre of the Republic of Srpska due to SARS-CoV2caused pneumonia, in whom hsTnT was measured, were included. The Kaplan-Meier analysis was used to assess differences in all-cause mortality between the groups. Independent predictors of all-cause mortality were identified through univariateand multivariate Cox regression analysis.

Results: Compared with patients who had normal hsTnT levels, patients with raised hsTnT were significantly older ( $70.7 \pm 13.23$ vs $49 \pm 15.29$; $p<0.001)$. Glucose values were significantly increased in patients with raised hsTnT $(9.29 \pm 5.14$ vs $6.76 \pm 2.46$ [4.1-5.9] mmol/L; $\mathrm{p}=0.005)$, as well as serum creatinine (179.07 \pm 225.58 vs 87.53 $\pm 18.16 \mu \mathrm{mol} / \mathrm{L} ; \mathrm{p}=0.01)$, hsTnT $(187.43 \pm 387.29$ vs $7.58 \pm 3.40 \mathrm{pg} / \mathrm{mL} ; \mathrm{p}=0.003)$, D-dimer (5.94 \pm 13.78 vs $1.04 \pm 1.26$ [0-0.50] mg/L; $\mathrm{p}=0.024$ ), C-reactive protein $(125.92 \pm 116.82$ vs $69.97 \pm 73.09)[<5.0] \mathrm{mg} / \mathrm{L} ; \mathrm{p}=0.009)$ and calcium $(1.32 \pm 0.46$ vs $1.03 \pm 0.173$ [2.20-2.65] mmol/L; $\mathrm{p}=0.001$ ). Kaplan-Meier analysis revealed that the number of all-cause deaths at 1 year was 19 of whom 18 were presented with elevated hsTnT (log-rank $\mathrm{p}<0.001$ ). When univariate Cox regression was applied, multiple predictors of all-cause mortality have been identified ie age, haemoglobin, haematocrit, urea, CK-MB as well as hsTnT. In a multiple regression model, hsTnT remained an independent predictor of poor outcome.

Conclusion: Results from this study showed that the value of hsTnT during hospitalisation is possibly associated with long-term poor outcome of COVID-19 patients. Therefore, hsTnT may appear as a surrogate factor to differentiate between patients at high risk who need more intensive follow-ups.
\end{abstract}

Key words: COVID-19; High-sensitive Troponin-T; All-cause mortality.
(1) Medical Faculty, University of Banja Luka, Bosnia and Herzegovina.

(2) Department of Cardiology, University Clinical Centre of the Republic of Srpska, Banja Luka, Bosnia and Herzegovina.

(3) Department of Pulmonology, University Clinical Centre of the Republic of Srpska, Banja Luka, Bosnia and Herzegovina.

Correspondence: BOJAN STANETIĆ

E: bojan.stanetic@med.unibl.org

\section{ARTICLE INFO}

Received: 8 April 2021

Revision received: 14 May 2021 Accepted: 15 May 2021

\section{Introduction}

Since December 2019, the humanity is constantly under affection of Severe acute respiratory syndrome coronavirus 2 (SARS-CoV-2), which caus- es illness known as Coronavirus disease 2019 (COVID-19). As the first cases were recorded in Wuhan, Hubei Province, China no one assumed it

Copyright $\odot 2021$ Stojanović et al. This is an open access article distributed under the Creative Commons Attribution License (CC BY), which permits unrestricted use, distribution, and reproduction in any medium, provided the original work is properly cited. This article should be cited as follows: Stojanović D, Ćetojević Ž, Dujaković B, Stanetić M, Kovačević-Preradović T, Stanetić B. High-sensitive Troponin-T as a predictive outcome factor of COVID-19 hospitalised patients: analysis after one-year follow-up. Scr Med 2021 Jun;52(2):96-103. 
would have impact in global proportions. The new virus has caused a lot of repercussions in all segments and spheres of human lives, such as health issues, economic consequences and changes in global politics. All over the globe, the new virus has spread onto (over) 223 countries (areas, territories) and infected $120,164,106$ people to this day. Due to absence of specific treatment, a total of 2,660,422 people died because of infection, and these numbers continue to rise every day. ${ }^{1}$ In the Republic of Srpska, first case of SARS-CoV-2 was registered on 5 March 2020. Since then, there were 47,993 confirmed cases, from which 2,420 people died, and 37,342 have successfully recovered. ${ }^{2}$

Until now, many impacts of SARS-CoV-2 to the human body were found. ${ }^{1}$ Infection can be asymptomatic, it may have mild, moderate or severe symptoms, or it could have symptoms of critical illness (acute respiratory distress syndrome, respiratory failure, shock or multiorgan system dysfunction). Respiratory symptoms are the main manifestation of COVID-19 infection, but the evidence also indicates affection of many other systems: cardiovascular, gastrointestinal, nervous, as well as renal, endocrine and haematological manifestations. ${ }^{1}$ The heart symptomatology varies, starting with increased serum levels of heart-specific enzymes, over various kinds of arrhythmias all the way to myocardial injury. There were also reported isolated cases of COVID-19-induced myocarditis, probably caused by direct myocardial injury of SARS-CoV-2. The fact that angiotensin-converting enzyme 2, the receptor SARSCoV-2 uses, is significantly increased in cardiac tissue, support this thesis. ${ }^{3}$ One study found that $16.7 \%$ COVID-19 infected patients suffered from arrhythmias, while $7.2 \%$ had severe myocardial injury. ${ }^{4}$

Despite global dissemination, neither the treatment or the specific predictive factors (especially in a long-term) have been found or strictly defined yet. Therefore, in order to establish correlation between specific parameters and organ systems (cardiovascular primarily) it was decided to gather a group of COVID-19 affected patients and did a follow-up study during the time of 365 days (1 year).

\section{Methods}

\section{Study population}

Between March 5, 2020 and March 31, 2020, consecutive patients hospitalised at University Clinical Centre of the Republic of Srpska due to SARSCoV2-caused pneumonia, in whom high-sensitive Troponin-T (hsTnT) was measured, were included (Figure 1). The study group comprised of patients with polymerase-chain-reaction (PCR)-confirmed infection only. The study was approved by the local ethics committee. Since this was an observational (ie, non-interventional) study, informed consent had been waived.

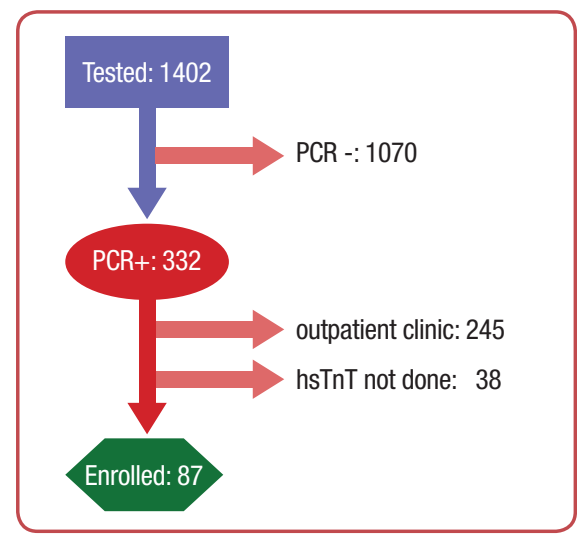

Figure 1: Study flow chart

\section{Data collection}

Data acquisition consisted of separate entries for classical factors of affected person history, cardiovascular threat factors, laboratory exams and data on long-term 1-year follow-up. Laboratory values, which included hsTnT have been collected at admission as well as during hospitalisation. Peak values for hsTnT were used for calculation. Electrocardiogram (ECG) was not routinely recorded due to logistic reasons. Follow-ups have been retrieved from electronic and written records, as well as by phone contacts.

Patients were divided into two groups ie troponin-negative (hsTnT < 14) and troponin - positive (hsTnT $\geq 14$ ). During the one-year follow-up of the initial presentation, data on all-cause death were acquired.

\section{Statistical analysis}

Statistical analysis was performed using SPSS (Version 25; MAC). Descriptive statistics were given as mean plus standard deviation or median plus interquartile range, based on the distribution, which was tested by Kolmogorov-Smirnov test. 
Categorical variables were presented as a number with a percentage and tested by Chi-squared test. Differences between means were assessed by the Student's t-test when normally distributed ie, Mann Whitney U-test when normal distribution could not be found. Kaplan-Meier analysis was used to assess differences in all-cause mortality between the groups. Independent predictors of mortality were identified through univariate- and multivariate Cox regression analysis.

\section{Results}

\section{Patient characteristics}

A total of 87 patients were included in this study, 44 of them (50.5\%) with raised hsTnT, and 43 $(49.4 \%)$ had normal hsTnT values. The medi- an age of patients was 63 years (range, $22-88$ years). Compared with patients who had normal hsTnT levels, patients with raised hsTnT were significantly older (70.7 $( \pm 13.23)$ vs 49 ( \pm 15.29$)$; $p$ $<0.001$ ). Among these patients, fever (67 patients or $77 \%$ ) was the most common symptom. Cough, shortness of breath, fatigue and muscle pain were present in 44 patients ( $50.6 \%), 39$ patients (44.8 $\%), 51$ patients $(58.6 \%)$ and 33 patients (37.9 $\%)$, respectively (Table 1 ). Headache (8 patients [9.2\%]), nausea (8 patients [9.2\%]) and diarrhoea (16 patients [18.4\%]) were rare. The most common coexisting conditions were diabetes (22 patients [25.3\%]) and hypertension (46 patients [52.9\%]). Six patients $(6.9 \%)$ had central nervous system disease, 2 (2.3\%) had liver disease, 9 patients $(10.3 \%)$ had renal disease and 8 patients $(9.2 \%)$ had problems with respiratory system. Twenty-seven patients (31\%) had heart issues, 10 of them ischaemic $(11.5 \%)$ and 11 of them $(12.6 \%)$ had some form of cardiomyopathy (Table 1).

Table 1: Baseline characteristics of hospitalised patients positive for COVID-19 related to hsTnT values

\begin{tabular}{|c|c|c|c|c|}
\hline Variable & hsTNT $\leq 14.0$ & hsTNT > 14.0 & p-value & Total \\
\hline \multicolumn{5}{|l|}{ Gender } \\
\hline Male & 34 & 29 & \multirow[t]{2}{*}{0.170} & 63 \\
\hline Female & 9 & 15 & & 24 \\
\hline \multicolumn{5}{|l|}{ Age } \\
\hline $0-50$ & 19 & 4 & \multirow[t]{2}{*}{0.000} & 23 \\
\hline$>50$ & 24 & 40 & & 64 \\
\hline \multicolumn{5}{|l|}{ Diabetes mellitus } \\
\hline No & 37 & 28 & \multirow[t]{2}{*}{0.016} & 65 \\
\hline Yes & 6 & 16 & & 22 \\
\hline \multicolumn{5}{|l|}{ Dyslipidaemia } \\
\hline No & 41 & 39 & \multirow[t]{2}{*}{0.250} & 80 \\
\hline Yes & 2 & 5 & & 7 \\
\hline \multicolumn{5}{|l|}{ Arterial hypertension } \\
\hline No & 31 & 10 & \multirow[t]{2}{*}{0.000} & 41 \\
\hline Yes & 12 & 34 & & 46 \\
\hline \multicolumn{5}{|l|}{ CNS disease } \\
\hline No & 40 & 41 & \multirow[t]{2}{*}{0.977} & 81 \\
\hline Yes & 3 & 3 & & 6 \\
\hline \multicolumn{5}{|l|}{ Liver disease } \\
\hline No & 43 & 42 & \multirow[t]{2}{*}{0.157} & 85 \\
\hline Yes & 0 & 2 & & 2 \\
\hline \multicolumn{5}{|l|}{ Kidney disease } \\
\hline No & 41 & 37 & \multirow{4}{*}{0.158} & 78 \\
\hline Acute renal failure & 0 & 1 & & 1 \\
\hline Chronic renal failure & 0 & 4 & & 4 \\
\hline Other & 2 & 2 & & 4 \\
\hline \multicolumn{5}{|l|}{ Lung disease } \\
\hline No & 41 & 38 & \multirow{3}{*}{0.250} & 79 \\
\hline Obstructive lung disease & 1 & 5 & & 6 \\
\hline Other & 1 & 1 & & 2 \\
\hline \multicolumn{5}{|l|}{ Heart disease } \\
\hline No & 41 & 19 & \multirow{5}{*}{0.000} & 60 \\
\hline Ischemic heart disease & 2 & 8 & & 10 \\
\hline Valvular heart disease & 0 & 3 & & 3 \\
\hline Cardiomyopathy & 0 & 11 & & 11 \\
\hline Other & 0 & 3 & & 3 \\
\hline
\end{tabular}

\begin{tabular}{|c|c|c|c|c|}
\hline Variable & hsTNT $\leq 14.0$ & hsTNT > 14.0 & p-value & Total \\
\hline \multicolumn{5}{|l|}{ Carcinoma(s) } \\
\hline No & 43 & 37 & \multirow[t]{2}{*}{0.006} & 80 \\
\hline Yes & 0 & 7 & & 7 \\
\hline \multicolumn{5}{|l|}{ Fever } \\
\hline No & 3 & 9 & \multirow[t]{2}{*}{0.034} & 12 \\
\hline Yes & 39 & 28 & & 67 \\
\hline \multicolumn{5}{|l|}{ Cough } \\
\hline No & 12 & 16 & \multirow[t]{2}{*}{0.334} & 28 \\
\hline Yes & 24 & 20 & & 44 \\
\hline \multicolumn{5}{|c|}{ Shortness of breath } \\
\hline No & 24 & 16 & \multirow[t]{2}{*}{0.092} & 40 \\
\hline Yes & 16 & 23 & & 39 \\
\hline \multicolumn{5}{|l|}{ Fatigue } \\
\hline No & 15 & 13 & \multirow[t]{2}{*}{0.580} & 28 \\
\hline Yes & 24 & 27 & & 51 \\
\hline \multicolumn{5}{|l|}{ Muscle pain } \\
\hline No & 20 & 21 & \multirow[t]{2}{*}{0.451} & 41 \\
\hline Yes & 19 & 14 & & 33 \\
\hline \multicolumn{5}{|l|}{ Headache } \\
\hline No & 30 & 28 & \multirow[t]{2}{*}{0.567} & 58 \\
\hline Yes & 5 & 3 & & 8 \\
\hline \multicolumn{5}{|l|}{ Nausea } \\
\hline No & 35 & 32 & \multirow[t]{2}{*}{0.905} & 67 \\
\hline Yes & 4 & 4 & & 8 \\
\hline \multicolumn{5}{|l|}{ Diarrhoea } \\
\hline No & 35 & 28 & \multirow[t]{2}{*}{0.197} & 63 \\
\hline Yes & 6 & 10 & & 16 \\
\hline \multicolumn{5}{|l|}{ Pneumonia } \\
\hline No & 3 & 5 & \multirow{3}{*}{0.329} & 8 \\
\hline Unilateral & 7 & 3 & & 10 \\
\hline Bilateral & 32 & 35 & & 67 \\
\hline
\end{tabular}

hsTnT: high-sensitive Troponin-T, ref values: < $14.0 \mathrm{pg} / \mathrm{mL}$; red colour: statistical significance; 
Table 2: Laboratory findings of hospitalised patients positive for COVID-19 related to hsTnT values

\begin{tabular}{|c|c|c|c|}
\hline Variable & hsTNT $\leq 14.0$ & hsTNT > 14.0 & $\mathrm{p}$-value \\
\hline Gender & $58.33 \pm 16.80$ & $65.91 \pm 19.08$ & 0.074 \\
\hline Age & $49 \pm 15.29$ & $70.7 \pm 13.23$ & 0.000 \\
\hline Erythrocytes $\times 10^{12}$ (per L) & $4.76 \pm 0.65$ & $4.12 \pm 1.21$ & $<0.001$ \\
\hline Haemoglobin (g/L) & $141.58 \pm 13.42$ & $120.48 \pm 2.89$ & $<0.001$ \\
\hline Haematocrit (L/L) & $0.43 \pm 0.05$ & $0.375 \pm 0.1$ & $<0.001$ \\
\hline Platelets $\times 10^{9}$ (per L) & $201.16 \pm 70.73$ & $245.09 \pm 110.45$ & 0.030 \\
\hline Leukocytes $\times 10^{9}$ (per L) & $6.41 \pm 2.62$ & $9.44 \pm 5.46$ & 0.002 \\
\hline Neutrophils (\%) & $70.0 \pm 17.0$ & $77.0 \pm 22.25$ & 0.076 \\
\hline Lymphocytes (\%) & $18.86 \pm 9.66$ & $13.73 \pm 9.25$ & 0.013 \\
\hline Monocytes (\%) & $10.33 \pm 15.19$ & $7.02 \pm 4.45$ & 0.170 \\
\hline Eosinophils (\%) & $0.55 \pm 0.98$ & $0.88 \pm 1.67$ & 0.270 \\
\hline Basophils (\%) & $0.14 \pm 0.355$ & $0.16 \pm 0.370$ & 0.860 \\
\hline Glucose (mmol/L) & $6.76 \pm 2.46$ & $9.29 \pm 5.14$ & 0.005 \\
\hline BUN (mmol/L) & $5.06 \pm 1.69$ & $2.01 \pm 8.56$ & $<0.001$ \\
\hline Creatinine ( $\mu \mathrm{mol} / \mathrm{L})$ & $88.0 \pm 22.0$ & $115.0 \pm 85.0$ & $<0.001$ \\
\hline AST (U/L) & $45.91 \pm 27.39$ & $55.73 \pm 55.18$ & 0.303 \\
\hline ALT (U/L) & $51.16 \pm 45.15$ & $54.08 \pm 63.53$ & 0.810 \\
\hline GGT (U/L) & $76.73 \pm 101.32$ & $115.58 \pm 100.73$ & 0.274 \\
\hline $\mathrm{LDH}(\mathrm{U} / \mathrm{L})$ & $307.77 \pm 134.64$ & $368.20 \pm 154.99$ & 0.060 \\
\hline CK (U/L) & $353.40 \pm 488.97$ & $394.63 \pm 602.18$ & 0.728 \\
\hline CK-MB (U/L) & $22.28 \pm 15.42$ & $29.13 \pm 18.68$ & 0.101 \\
\hline HsTnT (pg/mL) & $7.58 \pm 3.40$ & $187.43 \pm 387.29$ & 0.003 \\
\hline Feritin $(\mu \mathrm{g} / \mathrm{L})$ & $1413.92 \pm 1797.97$ & $2253.37 \pm 4293.38$ & 0.380 \\
\hline D-dimer ( $\mu \mathrm{g} / \mathrm{L})$ & $1.04 \pm 1.26$ & $5.94 \pm 13.78$ & 0.024 \\
\hline $\mathrm{CRP}(\mathrm{mg} / \mathrm{L})$ & $69.97 \pm 73.09$ & $125.92 \pm 116.82$ & 0.009 \\
\hline PCT (ng/mL) & $0.308 \pm 0.73$ & $6.53 \pm 28.37$ & 0.333 \\
\hline Sodium (mEq/L) & $139.30 \pm 2.82$ & $139.40 \pm 5.46$ & 0.914 \\
\hline Potassium (mmol/L) & $4.49 \pm 0.59$ & $4.65 \pm 0.75$ & 0.281 \\
\hline Calcium (mmol/L) & $1.03 \pm 0.173$ & $1.32 \pm 0.46$ & 0.001 \\
\hline Chloride (mmol/L) & $107.56 \pm 6.73$ & $107.69 \pm 8.64$ & 0.937 \\
\hline Lactate (mmol/L) & $3.38 \pm 1.07$ & $3.69 \pm 1.20$ & 0.253 \\
\hline
\end{tabular}

${ }^{*} B U N$, blood urea nitrogen; AST, aspartate aminotransferase; $A L T$, alanine aminotransferase; GGT, gamma-glutamyltransferase; $L D H$, lactate dehydrogenase; CK, creatine kinase; CK-MB, creatine kinase - myocardial band; hsTnT, high sensitive troponin T; CRP, C-reactive protein; PCT, procalcitonin; red colour: statistical significance;

\section{Laboratory findings}

Compared with patients who had normal hsTnT levels (Table 2), patients with raised hsTnT had lower red blood cell count (average, $4.11 \pm 0.77$ ) vs $4.83 \pm 0.49$ [4.00-6.3 $\left.\times 10^{12}\right]$ cells/L; $\mathrm{p}<0.001$ ), haemoglobin count $(120.48( \pm 2.89)$ vs 141.58 $( \pm 13.42)[120-180] \mathrm{g} / \mathrm{L} ; \mathrm{p}<0.001)$, haematocrit count $(0.37 \pm 0.065)$ vs $0.42 \pm 0.039)$ [0.40-0.52] $\mathrm{L} / \mathrm{L} ; \mathrm{p}<0.001)$, higher platelet count (245.09 \pm 110.45 vs $201.16 \pm 70.73$ ) [140-440 x $10^{9}$ ] cells/L; $\mathrm{p}=0.03)$ and higher white blood cell count ( 9.44 \pm 5.46 vs $6.41 \pm 2.62\left[4.0-10.0 \times 10^{9}\right]$ cells $/ \mathrm{L} ; \mathrm{p}=$ $0.002)$, but lower lymphocyte count $(13.73 \pm 9.25$ vs $18.86 \pm 9.66$ ) [20.0-45.0] \%; $p=0.013$ ); other leucocyte fractions did not differ significantly (neutrophils, monocytes, eosinophils, basophils). Glucose values were significantly increased in patients with raised hsTnT $(9.29 \pm 5.14$ vs $6.76 \pm$ 2.46 [4.1-5.9] mmol/L; $\mathrm{p}=0.005$ ), as well as creatinine $(179.07 \pm 225.58$ vs $87.53 \pm 18.16)$ [59104] $\mu \mathrm{mol} / \mathrm{L} ; \mathrm{p}=0.01)$, hsTnT $(187.43 \pm 387.29 \mathrm{vs}$

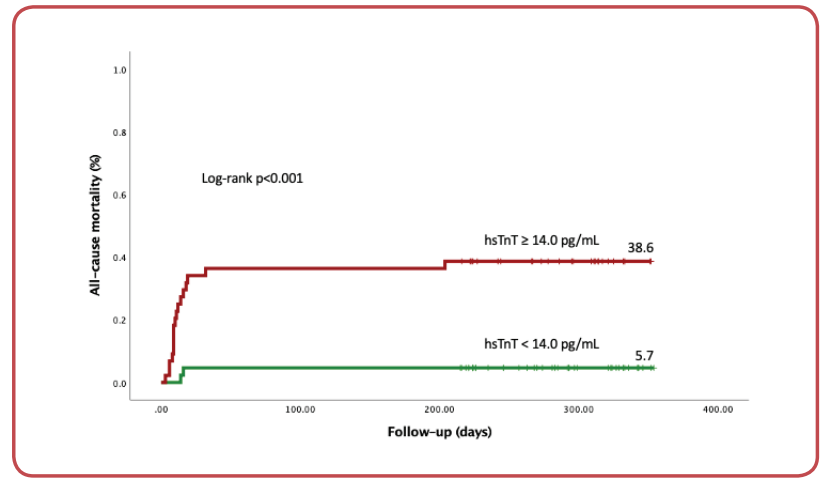

Figure 2: Kaplan-Meier analysis. The cumulative incidence rates of all-cause death between patients with and without elevated high-sensitive Troponin T at 1-year follow-up

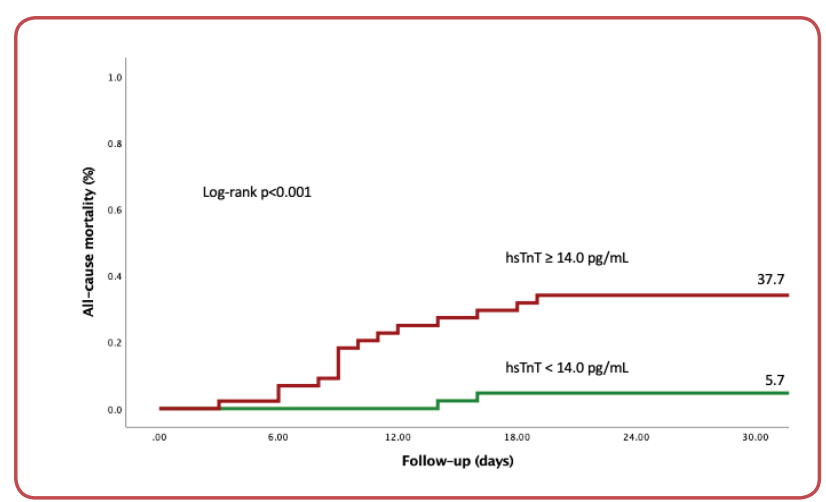

Figure 3: Kaplan-Meier analysis. The cumulative incidence rates of all-cause death between patients with and without elevated high-sensitive Troponin T at 30-days follow-up

$7.58( \pm 3.40)[\mathrm{pg} / \mathrm{mL}] ; \mathrm{p}=0.003)$, D-dimer values $(5.94 \pm 13.78)$ vs $1.04 \pm 1.26[0-0.50] \mathrm{mg} / \mathrm{L} ; \mathrm{p}=$ $0.024)$, C-reactive protein levels (125.92 \pm 116.82 vs $69.97 \pm 73.09[<5.0] \mathrm{mg} / \mathrm{L} ; \mathrm{p}=0.009)$ and calcium levels (1.32 \pm 0.46 vs $1.03 \pm 0.173$ ) [2.20-2.65] $\mathrm{mmol} / \mathrm{L} ; \mathrm{p}=0.001$ ). The liver enzymes (aspartate-aminotransferase, alanine-aminotransferase, gamma-glutamyl transferase) as well as other heart-specific enzymes (lactate-dehydrogenase, creatine-kinase, creatine-kinase myocardial band, lactate), ferritin, electrolytes (sodium, potassium, calcium and chloride) - showed no significant difference between the target groups.

\section{All-cause mortality and predictors analysis}

The number of all-cause deaths at 1 year was 19 of whom 18 were presented with elevated hsTnT (log-rank $\mathrm{p}<0.001$; Figure 2). The vast majority of deaths occurred in the first 30 days (Figure 3). When univariate logistic regression was applied (Table 3), multiple predictors of all-cause mortality have been identified, ie age $(p=0.003)$, haemoglobin ( $p=0.007)$, haematocrit ( $p=0.017)$, urea $(\mathrm{p}=0.030)$, CK-MB ( $\mathrm{p}=0.001)$ as well as hsTnT $(\mathrm{p}=0.002)$. In a multiple regression model, hsTnT was identified as an independent predictor 
Table 3: Univariate and multivariate Cox regression analysis of mortality predictors of hospitalised patients positive for COVID-19

\begin{tabular}{|c|c|c|c|c|c|c|}
\hline \multirow{2}{*}{ Variable } & \multicolumn{3}{|c|}{ Univariate regression } & \multicolumn{3}{|c|}{ Multivariate regression } \\
\hline & $\mathrm{HR}$ & Cl $95 \%$ & $p$ & $\mathrm{HR}$ & Cl $95 \%$ & $p$ \\
\hline Gender & 1.223 & $0.465-3.217$ & 0.684 & & & \\
\hline Age & 1.059 & $1.020-1.099$ & 0.003 & 1.047 & $0.958-1.143$ & 0.314 \\
\hline Erythrocytes & 0.453 & $0.255-0.804$ & 0.007 & 1.842 & $0.105-32.212$ & 0.676 \\
\hline Haemoglobin & 0.976 & $0.956-0.995$ & 0.015 & 1.014 & $0.861-1.195$ & 0.868 \\
\hline Haematocrit & 0.000 & $0.000-0.210$ & 0.017 & 0.000 & $0.000-1.499 \mathrm{E}+15$ & 0.417 \\
\hline Platelets & 0.998 & $0.993-1.003$ & 0.476 & & & \\
\hline Leukocytes & 1.000 & $0.906-1.103$ & 0.996 & & & \\
\hline Neutrophils & 1.002 & $0.979-1.026$ & 0.857 & & & \\
\hline Lymphocytes & 0.969 & $0.922-1.019$ & 0.222 & & & \\
\hline Monocytes & 0.926 & $0.831-1.031$ & 0.162 & & & \\
\hline Eosinophils & 0.814 & $0.516-1.284$ & 0.375 & & & \\
\hline Basophils & 0.038 & $0.000-13.445$ & 0.274 & & & \\
\hline Glucose & 1.052 & $0.966-1.146$ & 0.242 & & & \\
\hline BUN & 1.049 & $1.005-1.095$ & 0.030 & 0.880 & $0.731-1.060$ & 0.178 \\
\hline Creatinine & 1.001 & $0.999-1.003$ & 0.514 & & & \\
\hline AST & 0.991 & $0.975-1.007$ & 0.266 & & & \\
\hline ALT & 0.994 & $0.982-1.006$ & 0.348 & & & \\
\hline $\mathrm{LDH}$ & 1.001 & $0.999-1.004$ & 0.339 & & & \\
\hline GGT & 1.003 & $0.999-1.008$ & 0.155 & & & \\
\hline CK & 1.000 & $0.999-1.001$ & 0.722 & & & \\
\hline CK-MB & 1.035 & $1.015-1.056$ & 0.001 & 1.055 & $1.024-1.087$ & 0.000 \\
\hline hsTnT & 10.189 & 2.351-44.147 & 0.002 & 1.621 & $1.202-1.822$ & 0.001 \\
\hline Feritine & 1.000 & $1.000-1.000$ & 0.100 & & & \\
\hline D-dimer & 1.001 & $0.960-1.043$ & 0.975 & & & \\
\hline CRP & 0.999 & $0.994-1.004$ & 0.690 & & & \\
\hline PCT & 0.991 & $0.948-1.037$ & 0.708 & & & \\
\hline Sodium & 0.988 & $0.886-1.103$ & 0.833 & & & \\
\hline Potassium & 1.186 & $0.613-2.293$ & 0.613 & & & \\
\hline Calcium & 1.650 & $0.601-4.532$ & 0.331 & & & \\
\hline Chloride & 1.011 & $0.950-1.075$ & 0.739 & & & \\
\hline Lactate & 1.300 & $0.909-1.860$ & 0.150 & & & \\
\hline Diabetes mellitus & 1.363 & $0.518-3.586$ & 0.531 & & & \\
\hline Arterial hypertension & 3.864 & $1.282-11-650$ & 0.016 & 0.682 & $0.069-6.767$ & 0.744 \\
\hline Dyslipidaemia & 0.618 & $0.082-4.627$ & 0.639 & & & \\
\hline Heart disease & 1.703 & $1.283-2.260$ & 0.000 & 1.127 & $0.671-1.895$ & 0.651 \\
\hline CNS disease & 3.657 & $1.061-12.599$ & 0.040 & 992.742 & $21.287-46298.101$ & 0.000 \\
\hline Kidney disease & 1.075 & $0.757-1.527$ & 0.687 & & & \\
\hline Pulmonary disease & 1.490 & $0.816-2.722$ & 0.194 & & & \\
\hline Liver disease & 2.753 & $0.366-20.698$ & 0.325 & & & \\
\hline Cancer & 3.784 & $1.251-11.452$ & 0.018 & 3.300 & $0.469-23.193$ & 0.230 \\
\hline Fever & 0.225 & $0.087-0.583$ & 0.002 & 0.400 & $0.083-1.919$ & 0.252 \\
\hline Cough & 0.864 & $0.300-2.491$ & 0.787 & & & \\
\hline Shortness of breath & 2.253 & $0.845-6.003$ & 0.104 & & & \\
\hline Fatigue & 1.702 & $0.613-4.726$ & 0.308 & & & \\
\hline Muscle pain & 0.708 & $0.257-1.949$ & 0.504 & & & \\
\hline Headache & 2.221 & $0.610-8.087$ & 0.226 & & & \\
\hline Nausea & 0.565 & $0.074-4.294$ & 0.581 & & & \\
\hline Diarrhoea & 0.555 & $0.126-2.444$ & 0.437 & & & \\
\hline Pneumonia & 1.407 & 0.596-3.319 & 0.436 & & & \\
\hline
\end{tabular}




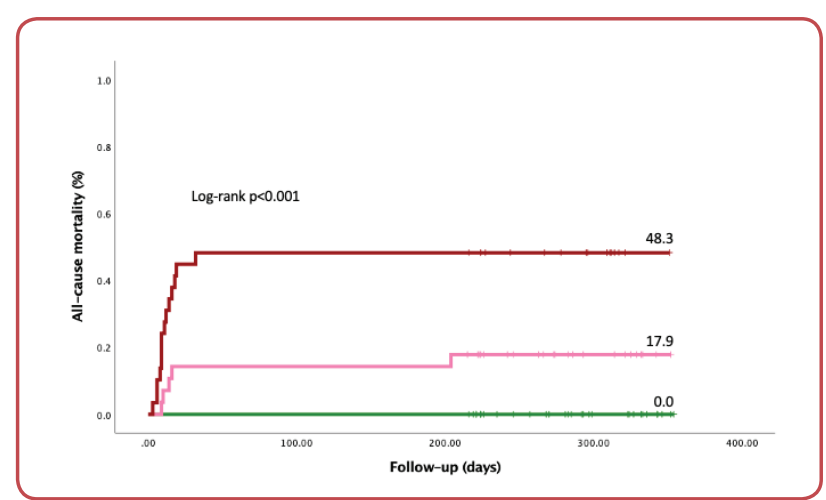

Figure 4: Kaplan-Meier analysis. The cumulative incidence rates of all-cause death stratified by high-sensitive Troponin $T$ terciles at 1-year follow-up

of poor outcome. In addition, the value of hsTnT correlated well with incidence of mortality (Kaplan-Meier analysis by hsTnT terciles - Figure 4).

\section{Discussion}

To our best knowledge, this study represented first report with follow-up of 1 year. It was shown that correlation exists between elevated hsTnT value and mortality in patients with COVID-19. After 1 year of hospitalisation, the value of hsTnT higher or equal than $14.0 \mathrm{pg} / \mathrm{mL}$ was associated with high risk of mortality in the studied population.

Cardiac troponin is one of the most important biomarkers in indication of cardiac injury. The troponin complex regulates the contraction of striated muscles and consists of three subunits - troponin C, troponin $\mathrm{T}$ and troponin I. In this region, troponin $\mathrm{T}$ is mostly used in diagnosis of cardiac injury. After myocardial injury, troponin starts to rise in peripheral blood within three to four hours and stays increased for ten to fourteen days. The result of modification of the fourth generation of cardiac assay is the new highly sensitive troponin, which is capable of detecting more subtle elevations indicative of cardiac injury. The normal range for high-sensitive troponin $\mathrm{T}$ in peripheral blood is up to $14 \mathrm{pg} / \mathrm{mL}^{.}{ }^{5}$ The role of troponin in COVID-19 in uncertain. In the Mayo clinic analysis of 367 patients, elevations of hsTnT were in $95 \%$ due to isolated myocardial injury. Most increases are due to either chronic illness such as heart failure or renal disease, followed by acute aetiologies such as critical illness or sepsis. Myo- carditis was rare; it was suspected as a potential cause of myocardial injury in only 3 patients and the diagnosis was not proven with cardiac magnetic resonance or biopsy. ${ }^{6}$ But in the Mayo clinic study elevated hsTnT were not predictor of mortality, just of events. Another studies from China, however, showed significant impact of troponin on mortality. Multivariate Cox regression analysis on 1,717 patients from the Tongji Hospital and the Hubei Xinhua hospitals identified troponin I as an independent predictor of 30-day mortality. ${ }^{7}$ In-hospital mortality was 10-fold higher in hospitalised patients with COVID-19 in Wuhan with myocardial injury (high-sensitive cardiac troponin above the $99^{\text {th }}$-percentile upper reference limit) which may reflect illness severity due to myocardial oxygen supply-demand imbalance. ${ }^{8}$ In this study of 87 patients who were hospitalised a year ago in University Clinical Centre of Republic of Srpska, the value of hsTnT was monitored and it was tried to establish the correlation between its value and outcome of hospitalised patients. It was found that 44 out of $87(50.5 \%)$ hospitalised patients had hsTnT value higher than 14.0. The highest measured hsTnT was 2126 $\mathrm{pg} / \mathrm{mL}$ and the lowest was $3 \mathrm{pg} / \mathrm{mL}$. The number of death cases was 19, while 18 of them were presented with high value of hsTnT. This finding showed that the value of hsTnT is possibly associated with outcome of COVID-19 positive patients. The factor that contributes to high mortality and cardiac damage was age: out of 44 patients with elevated hsTnT, 40 of them were older than 50 years (Table 1$)$. It is a strong predictor in univariate regression model $(\mathrm{p}=0.003)$ (Table 3$)$. Among 46 patients with chronic arterial hypertension, 34 of them (73.9\%) had hsTnT values over $14.0 \mathrm{pg} / \mathrm{mL}$. Among 19 death outcomes, 15 of them (78.9\%) were presented with arterial hypertension and all of them had elevated hsTnT (the lowest 14.5 and the highest $2126 \mathrm{pg} / \mathrm{mL}$ ). Also, the study found that among 22 patients with diabetes, 6 had death outcome (27.2\%), and all 6 were presented with hsTnT levels over 14.0 (range between 18.6 - $2126 \mathrm{pg} / \mathrm{mL}$ ).

Based on many previous studies, angiotensin-converting enzyme $2\left(\mathrm{ACE}_{2}\right)$ is a human cell receptor with a strong binding affinity to the Spike protein of the SARS-CoV-2, and $\mathrm{ACE}_{2}$ is also highly expressed in heart, so it can be assumed that COVID-19-induced cardiac injury might be mediated by $\mathrm{ACE}_{2}{ }^{9}$ Autopsy report from other 
study showed interstitial mononuclear inflammatory infiltrates in heart tissue. Inflammatory biomarkers were significantly increased in patients with cardiovascular diseases, indicating that inflammatory cell necrosis promoted inflammatory response and led to cytokine storm damage to the myocardium, which can be severe and even lead to fulminant myocarditis. ${ }^{4}$ Moreover, the systemic inflammatory response may trigger rupture or erosion of coronary plaques in patients with underlying coronary artery disease. ${ }^{10,11}$ The overall mortality rate in this study was $21.8 \%$ (19 out of 87 patients). Similar findings were found in a meta-analysis of 12,262 patients from 13 studies where mortality rate was $23 \%$ (20-26\%). Elevated troponins were found in $31 \%$ (23-38\%) with clear association with increased mortality (odds ratio 4.75, CI 4.07-5.53; $\mathrm{p}<0.01$ ) suggesting that chance of mortality is $45 \%$ in patients with elevated troponin and 14 $\%$ in patients with non-elevated troponin. ${ }^{12}$ Interestingly enough, the correlation was not influenced by age $(p=0.218)$, male gender $(p=0.707)$, hypertension $(p=0.182)$, diabetes $(p=0.906)$ or coronary artery disease $(p=0.864)$.

Recently, in a meta-analysis of 51 studies, ${ }^{13}$ elevated troponin values were associated with a higher risk of subsequent death during hospitalisation (RR 2.68, 95 \% CI 2.08-3.46). The same analysis showed that after adjustment for confounders, troponin was an independent predictor of mortality in multivariable analysis. ${ }^{13}$ These results revealed similar predictive performance in a long-term follow-up (HR 1.621, $95 \%$ CI 1.202-1.822). Even more interesting is the fact that the meta-analysis found a post-test probability of death about $42 \%$ for patients with raised troponins on admission (in this analysis 18 out of 44 patients ( $40.9 \%$ ) with hsTnT $\geq 14$ died during follow-up). Another cohort study which included 1,053 patients with COVID-19 revealed that troponin I higher than $0.34 \mathrm{ng} / \mathrm{mL}$ was strong independent predictor of 1-month mortality (OR 4.38; $\mathrm{p}<0.001)$. Patients with a mortality score using hypoxia at admission, age, and troponin I elevation, age (HA2T2) $\geq 3$ had a 1-month mortality of $43.7 \%$ whereas the remaining population had mortality of $5.9 \%$ (AUC 0.834 ).

\section{Limitations}

This study has some limitations. Because of the observational and retrospective nature of the study, data collection may be incomplete. Some data were missing at the beginning of research, ie hypoxia measurements were not done in all patients. Furthermore, due to logistic reasons additional diagnostic tests were not routinely recorded to rule out STEMI or NSTEMI, ie ECG and echocardiogram. Since this was not a randomised trial, there was no possibility to exclude the potential selection bias. The sample size was small, however, follow-up at one year was collected.

\section{Conclusion}

1. Elevated values of highly sensitive troponins represent state of myocardial injury, which was not rare and strong predictor in COVID-19 patients.

2. There are multiple conditions, such as worsening of chronic ischaemia or acute inflammation, which can lead in troponin increase.

3. High-sensitive Troponin T may appear as a surrogate factor to differentiate between patients at high risk who need more intensive follow-up.

\section{Acknowledgements}

None.

\section{Conflict of interest}

None.

\section{References}

1. World Health Organisation. WHO. Coronavirus disease (COVID-19). [Internet] [Cited 17-Mar-2021]. Available at: https://www.who.int/emergencies/diseases/novel-coronavirus-2019.

2. IZJZRS. Institut za javno zdravstvo Republike Srpske. [Internet] [Cited: 18-Mar-2021]. Available at: phi.rs.ba. 
3. Gavriatopoulou M, Korompoki E, Fotiou D, Ntanasis-Stathopoulos I, Psaltopoulou T, Kastritis E, et al. Organ-specific manifestations of COVID-19 infection. Clin Exp Med 2020 Nov;20(4):493-506.

4. Li J, Guo T, Dong D, Zhang X, Chen X, Feng Y, et al. Defining heart disease risk for death in COVID-19 infection. QJM 2020 Dec 1;113(12):876-82.

5. Xu RY, Zhu XF, Yang Y, Ye P. High-sensitive cardiac troponin T. J Geriatr Cardiol 2013 Mar;10(1):102-9.

6. De Michieli L, Ola O, Knott JD, Akula A, Mehta RA, Hodge DO, et al. High-sensitivity cardiac troponin T for the detection of myocardial injury and risk stratification in COVID-19. Clin Chem 2021 Apr 16:hvab062. doi: 10.1093/ clinchem/hvab062.

7. Jiang M, Li C, Zheng L, Lv W, He Z, Cui X, et al. A biomarker-based age, biomarkers, clinical history, sex (ABCS)-mortality risk score for patients with coronavirus disease 2019. Ann Transl Med 2021 Feb;9(3):230.

8. Chapman AR, Bularga A, Mills NL. High-sensitivity cardiac troponin can be an ally in the fight against COVID-19. Circulation 2020 Jun 2;141(22):1733-5.

9. Yang $\mathrm{C}$, Jin $\mathrm{Z}$. An acute respiratory infection runs into the most common noncommunicable epidemic-COVID-19 and cardiovascular diseases. JAMA Cardiol 2020 Jul 1;5(7):743-4.
10. Zaim S, Chong JH, Sankaranarayanan V, Harky A. COVID-19 and multiorgan response. Curr Probl Cardiol 2020 Aug;45(8):100618.

11. Wang D, Hu B, Hu C, Zhu F, Liu X, Zhang J, et al. Clinical characteristics of 138 hospitalized patients with 2019 novel coronavirus-infected pneumonia in Wuhan, China. JAMA 2020 Mar 17;323(11):1061-9.

12. Wibowo A, Pranata R, Akbar MR, Purnomowati A, Martha JW. Prognostic performance of troponin in COVID-19: A diagnostic meta-analysis and meta-regression. J Infect Dis 2021 Apr;105:312-8.

13. Zhao BS, Liu WF, Lei SH, Zhou BW, Yang X, Huang TY, et al. Prevalence and prognostic value of elevated troponins in patients hospitalised for coronavirus disease 2019: a systematic review and meta-analysis. Journal of Intensive Care 2020;23(8):88.

14. Manocha KK, Kirzner J, Ying X, Yeo I, Peltzer B, Ang B, et al. Troponin and other biomarker levels and outcomes among patients hospitalized with COVID-19: derivation and validation of the HA2T2 COVID-19 mortality risk score. J Am Heart Assoc 2021 Mar 16;10(6):e018477. 